\title{
First detection and genetic characterization of ungulate tetraparvovirus 2 and ungulate tetraparvovirus 4 in special livestock on the Qinghai-Tibet Plateau in China
}

\author{
Yangyang Pan ${ }^{1}$, Yun Wang ${ }^{2}$, Meng Wang ${ }^{1}$, Qian Zhang ${ }^{1}$, Abdul Rasheed Baloch ${ }^{3}$, Jun Zhou ${ }^{4}$, Jing Ma ${ }^{4}$, Jam Kashif ${ }^{5}$,
} Gengquan Xu', Libin Wang ${ }^{1}$, Jiangfeng Fan ${ }^{1}$, Yan Cui ${ }^{1}$ and Sijiu Yu ${ }^{1 *}$

\begin{abstract}
Tetraparvovirus, formerly known as Partetravirus, is a newly discovered genus in the family Parvoviridae that is considered phylogenetically distinct from other parvoviruses. However, nothing is known about the prevalence of Tetraparvovirus in special livestock living on the Qinghai-Tibet Plateau of China, such as Tibetan pigs and Tibetan sheep. A pair of special primers was designed based on the conserved regions in the genome of ungulate tetraparvovirus 2 (P-PARV4) and ungulate tetraparvovirus 4 (O-PARV4) and was used to detect P-PARV4 in domestic pigs and Tibetan pigs and O-PARV4 in ovines and Tibetan sheep. The results showed a 15.59 and $9.38 \%$ prevalence of P-PARV4 in domestic pigs (18.96\% in Gansu Province and 11.76\% in Qinghai Province) and Tibetan pigs (14.28\% in Gansu Province and 4.44\% in Qinghai Province), respectively, and a 7.31 and 5.20\% prevalence of O-PARV4 in ovines (6.61\% in Gansu Province and 8.00\% in Qinghai Province) and Tibetan sheep (4.55\% in Gansu Province and $5.50 \%$ in Qinghai Province), respectively. The prevalence of P-PARV4 was 14.76\% (31/210) for $\leq 1$-month-old pigs and $10.58 \%(20 / 189)$ for $>1$-month-old pigs, and the positive rates of O-PARV4 were $7.65 \%$ (18/235) for $\leq 1$-monthold sheep and 5.05\% (11/218) for > 1-month-old sheep. The phylogenetic analysis of NS1, VP1, VP2 and the whole PARV4-related provirus genome demonstrated that both P-PARV4 and O-PARV4 sequences in this study were more closely related to the sequences of other strains discovered in the same genus of animals. The identity analyses for the full-length VP2 genomes of O-PARV4 revealed $98.84-100.00 \%$ sequence identity among the 7 strains and the previously reported strain, which was $98.60-99.28 \%$ for P-PARV4. In the present study, for the first time, we have provided comprehensive information regarding the widespread infection of P-PARV4 and O-PARV4 in special livestock on the Qinghai-Tibet Plateau in China. Our present findings highlight the importance of epidemiologic surveillance to limit the spread of Tetraparvovirus in livestock at high altitudes in China.
\end{abstract}

Keywords: Ungulate tetraparvovirus 2, Ungulate tetraparvovirus 4, Tibetan pig, Tibetan sheep, Qinghai-Tibet plateau

Members of the family Parvoviridae are small, non-enveloped viruses with single-stranded DNA genomes of approximately 5 kilobases $(\mathrm{kb})$ [1]. The genome of most parvoviruses contains two open reading frames (ORFs) separated by a small non-coding region, where ORF1 encodes non-structural proteins (NS) and ORF2 encodes two overlapping viral capsid proteins (VP1/VP2) [1]. The

\footnotetext{
*Correspondence: sijiuy@126.com; sjyu@163.com

${ }^{1}$ Gansu Agricultural University, College of Veterinary Medicine, Lanzhou 730070, China

Full list of author information is available at the end of the article
}

development of molecular biology-based detection methods led to the discovery that parvoviruses are widespread pathogens that cause a wide range of diseases in livestock and humans [2]. According to the International Committee on the Taxonomy of Viruses (ICTV) 2018, Tetraparvovirus (previously proposed as "Partetravirus") is a newly established genus in the Parvoviridae family that includes primate tetraparvovirus 1 (formerly known as human parvovirus 4, PARV-4) and six novel PARV4-related animal parvoviruses described in the past decade, viz., chiropteran tetraparvovirus 1, primate

(c) The Author(s). 2019 Open Access This article is distributed under the terms of the Creative Commons Attribution 4.0 International License (http://creativecommons.org/licenses/by/4.0/), which permits unrestricted use, distribution, and 
tetraparvovirus 1, ungulate tetraparvovirus 1 (bovine hokovirus 1, B-PARV4), ungulate tetraparvovirus 2 (porcine hokovirus, P-PARV4), ungulate tetraparvovirus 3 (porcine $\mathrm{Cn}$ virus, $\mathrm{CnP}-\mathrm{PARV} 4$ ) and ungulate tetraparvovirus 4 (ovine hokovirus, O-PARV4) [3]. The prevalence of P-PARV4 and B-PARV4 has been widely reported throughout the world, including Gansu and Qinghai Provinces in China [3, 4]. We also previously discovered B-PARV4 in domestic yaks (Bos grunniens) [3], which are also considered special livestock on the Qinghai-Tibet Plateau. However, only one report describes the members of the O-PARV4 species that was discovered in Hong Kong in 2011 [5]. To date, no other report of this novel parvovirus exists anywhere in the world. Here, for the first time, we report P-PARV4 and O-PARV4 infections in livestock from the Qinghai-Tibet Plateau in China.

The Qinghai-Tibet Plateau is an extensive pastoral and semi-pastoral area located in Asia, northeast of the Himalayas. It is the highest region on earth (altitudes $>3000 \mathrm{~m}$, average annual temperature $<0{ }^{\circ} \mathrm{C}$ ) and includes the Qinghai, Gansu Sichuan and Tibet Provinces in northwestern China. Yak, Tibetan pigs and Tibetan sheep are the main livestock that live on the Qinghai-Tibet Plateau in China. Because they live in a cold and harsh environment on a plateau for an extended period of time, the livestock in these areas have undergone specific selection to enrich disease resistance-related gene categories in their genome $[6,7]$. However, some pathogenic infections have been discovered in these livestock in recent years, such as hepatitis E virus (HEV) [8], Brucella spp. [9], bovine viral diarrhoea virus (BVDV) [10] and B-PARV4 [3] infections in yaks, swine influenza A virus (SIV) [11] and highly pathogenic porcine reproductive and respiratory syndrome virus (HP-PRRSV) [12] infections in Tibetan pigs, and the infection rates of these pathogens were lower than those in regular production animals [9-11]. All the data indicate that livestock on the Qinghai-Tibet Plateau in China are susceptible to pathogenic infections. P-PARV4 and O-PARV4 infections in China have been reported for many years. Additionally, the prevalence of B-PARV4 in yaks that were farmed together with Tibetan pigs and Tibetan sheep, which we have been involved in research on P-PARV4 and B-PARV4 and the surveillance of livestock infections on the Qinghai-Tibet Plateau. Thus, it is necessary to investigate and prevent P-PARV4 and O-PARV4 infections in the highland region in China.

Our present study aimed to investigate the distribution of P-PARV4 and O-PARV4 in domestic pigs, Tibetan pigs, ovines and Tibetan sheep on the Qinghai-Tibet Plateau in China based on the amplification of tetraparvovirus VP2 by polymerase chain reaction (PCR) using primers we designed based on the conserved regions in the VP2 sequences of P-PARV4 and O-PARV4. Full-length sequences of NS1, VP1, and VP2 and nearly full-length sequences of the identified PARV4-related viruses (seven strains from pigs, three from sheep) were also determined and analysed. The results should provide a solid foundation for the improved control of Tetraparvovirus infection in livestock in this region of China.

\section{Methods}

From May 2016 to June 2017, blood samples from 218 domestic pigs, 181 Tibetan pigs, 246 ovines and 219 Tibetan sheep were collected from Gansu and Qinghai Provinces surrounding the Qinghai-Tibet Plateau in China with the assistance of the Animal Health and Epidemiology Center and Veterinary Department (see Additional file 1). The samples were collected from apparently healthy animals. The specimens were grouped according to their species, ages and geographical region (see Additional file 1). Genomic DNA was extracted from $100 \mu \mathrm{L}$ of whole blood from each sample using the E-Z $96^{\circ}$ Blood DNA Kit (Omega, Norcross, GA, United States) according to the manufacturer's instructions. The extracted DNA was stored at $-20^{\circ} \mathrm{C}$ until required for PARV4-related detection and PCR amplification of full-length sequences.

To detect PARV4-related viruses in special livestock by PCR, one pair of special primers, PARV4-F and PARV4-R, was designed (see Additional file 2) using Primer Premier 6.24, targeting a 640-bp fragment of the conserved VP2 region based on multiple alignment of the reference P-PARV4 (EU200676) and O-PARV4 (JF504700) genomic sequences available in the National Center for Biotechnology Information (NCBI) GenBank database. PCR products were confirmed by $1.2 \%$ agarose gel stained with ethidium bromide and were visualized under ultraviolet light. DNA of P-PARV4 in domestic pigs from eastern China and sterilized water were used as the PCR positive and negative controls, respectively.

Seven P-PARV4-positive samples and eight O-PARV4positive samples were randomly selected based on region and species. Each of the 4 pairs of primers that produced overlapping PCR products was designed based on the reference P-PARV4 (EU200676) and O-PARV4 genomes (JF504700) (see Additional file 2), which were used to obtain the PARV4-related genome consisting of all the ORFs. The four different PARV4-related provirus genome PCR amplicons from each individual were purified using the QIAquick Gel Extraction Kit (Qiagen, Germany), cloned utilizing a TA cloning kit (TaKaRa TA Kit; Dalian, China) and subsequently transformed into competent Escherichia coli cells $(\mathrm{DH} 5 \alpha)$. Four purified recombinant plasmids were sequenced (Sangon Biotech, Shanghai, China), assembled, and edited by MEGA 7.1 [13] and DNAMAN 9.0 software to produce the final sequences of the viral genomes. Seven complete genomic sequences for P-PARV4 
(MG365914-MG365920) and eight sequences for O-PARV4 (MG365906-MG365913) were deposited in the GenBank database and used for further analysis. Full-length NS1, VP1, and VP2 sequences and nearly full-length sequences of seven P-PARV4 strains and eight O-PARV4 strains detected in this study were independently used in sequence alignment and phylogenetic and identity analyses with the B-PARV4, O-PARV4, and P-PARV4 sequences available in the NCBI GenBank database.

\section{Results}

The PARV4-related viruses were detected from 864 livestock blood samples by PCR targeting VP2 regions. For P-PARV4, the blood samples of domestic pigs and Tibetan pigs at two different ages were collected from Gansu and Qinghai Provinces. Positive samples for P-PARV4 in domestic pigs were found in 22 of 116 pigs (18.97\%) in Gansu Province and 12 of 102 pigs (11.76\%) in Qinghai Province. In Gansu Province, the positive rates of P-PARV4 in domestic pigs were 20.31\% (13/64) for $\leq 1$-month-old pigs and $17.31 \%(9 / 52)$ for > 1 -month-old pigs and were $13.56 \% \quad(8 / 59)$ for $\leq 1$-month-old pigs and 9.30\% (4/43) for >1-month-old pigs in Qinghai Province (see Additional file 1). In
Tibetan pigs, the positive rates of P-PARV4 in Gansu Province were $14.29 \%$ (13/91), including 16.33\% (8/49) for $\leq 1$-month-old pigs and $11.90 \%$ (5/42) for > 1 -month-old pigs, and were $4.44 \%(4 / 90)$ in Qinghai Province, including $5.26 \%$ (2/38) for $\leq 1$-month-old pigs and $3.85 \%(2 / 52)$ for $>1$-month-old pigs.

For O-PARV4, positive samples in Gansu Province were found in 8 of 121 ovines (6.61\%) and 5 of 110 (4.55\%) Tibetan sheep; additionally, the positive rates of P-PARV4 in ovines were $7.46 \%(5 / 67)$ for $\leq 1$-month-old ovines and $5.56 \%(3 / 54)$ for $>1$-month-old ovines, and they were $6.45 \%(4 / 62)$ for $\leq 1$-month-old ovines and 2.08\% (1/48) for $>1$-month-old ovines (see Additional file 1). In Qinghai Province, positive samples for O-PARV4 were found in 10 of 125 ovines $(8.00 \%)$ and 6 of 109 Tibetan sheep (5.50\%). The positive rates of O-PARV4 in ovines were $8.33 \%(6 / 72)$ for $\leq 1$-month-old ovines and $7.55 \%(4 / 53)$ for $>1$-month-old ovines, and they were $6.67 \%$ (3/45) for $\leq 1$-month-old ovines and 4.76\% (3/63) for $>1$-month-old ovines (see Additional file 1).

The phylogenetic trees based on the complete genome sequence and the NS1, VP1, and VP2 genes clearly showed three main branches, encompassing B-PARV4, O-PARV4, and P-PARV4. The seven new P-PARV4 genomes in domestic pigs and Tibetan pigs clustered on the same branch

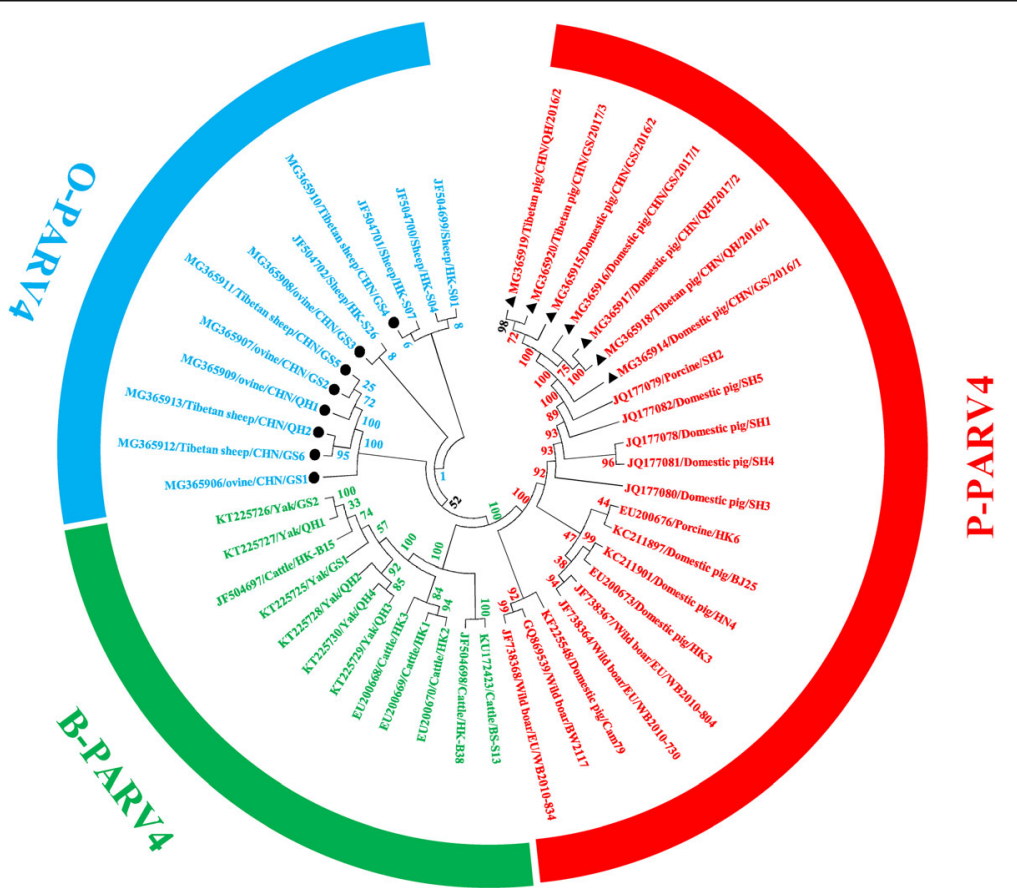

Fig. 1 Maximum likelihood (ML) phylogenetic tree constructed from complete PARV4-related genomic sequences. The ML phylogenetic tree was constructed using the complete PARV4-related genomic sequences from the seven P-PARV4 strains and eight O-PARV4 strains detected in this study (submitted to the GenBank nucleotide sequence database with accession numbers MG368906-MG368920) and 30 reference sequences obtained from the GenBank nucleotide sequence database. One thousand replications were performed to calculate bootstrap values (indicated on the tree). The strains identified in the phylogenetic tree are indicated by GenBank accession numbers and breeds and strains. The seven newly identified P-PARV4 strains and eight O-PARV4 strains described in the present study are indicated by " $\mathbf{\Delta}$ " and "•", respectively. P-PARV4, O-PARV4 and B-PARV4 strains are shown in red, blue and green around the circumference of the figure, respectively 
as all known P-PARV4 genomes. Additionally, the eight new O-PARV4 genomes in ovines and Tibetan sheep clustered on the same branch as all known O-PARV4 genomes (Figs. 1 and 2). On the phylogenetic tree based on the complete genome sequence of PARV4-related provirus (Fig. 1), the main branch of P-PARV4 was divided into three additional branches, and the seven new P-PARV4 strains in domestic pigs and Tibetan pigs were closely related to each other and formed one branch with the P-PARV4 strains isolated from domestic pigs in eastern China (JQ177078-JQ177082) (Fig. 1). The main branch of O-PARV4 strains was also divided into three further
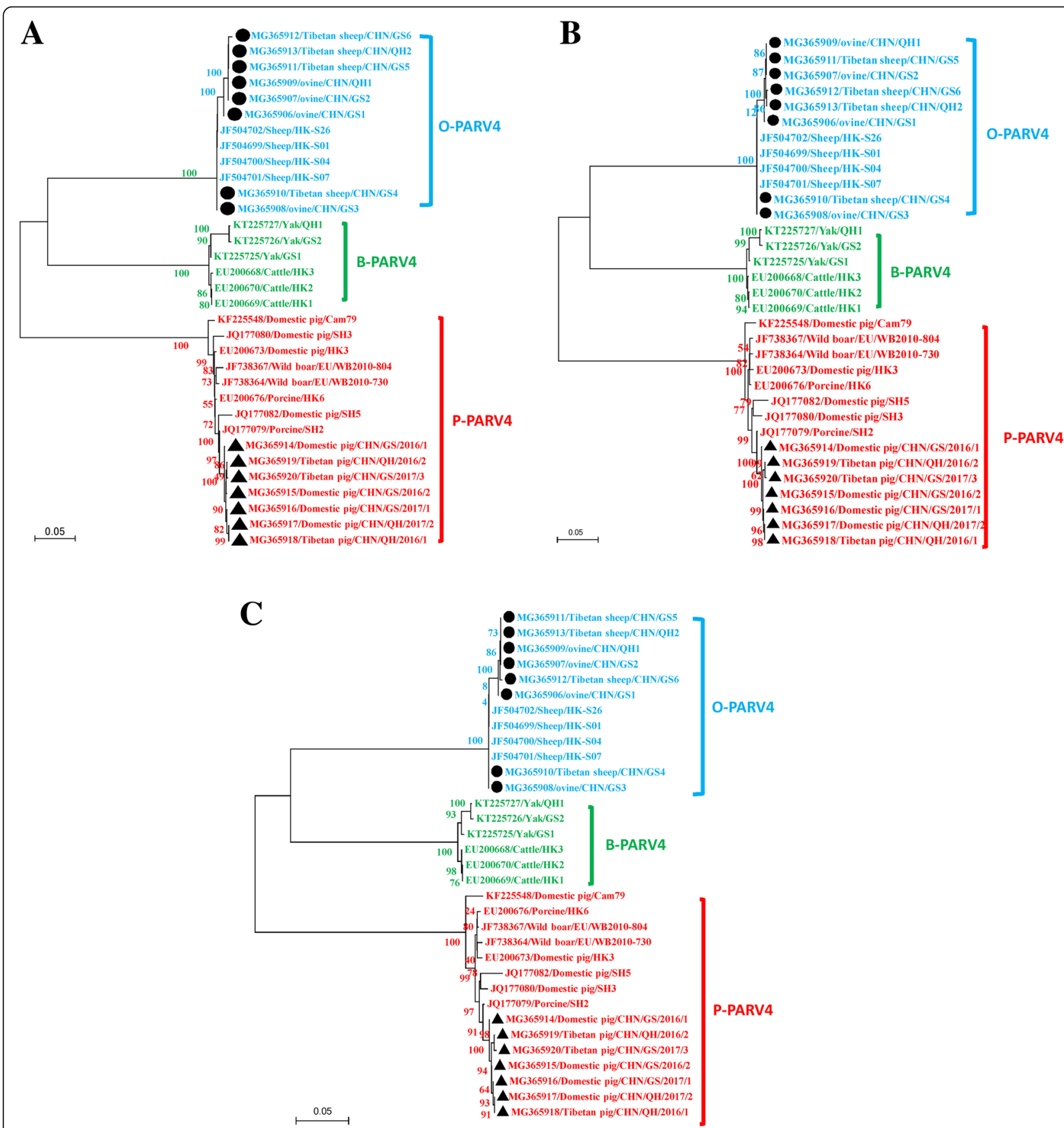

Fig. 2 Phylogenetic analysis of PARV4-related viruses based on NS1(A), VP1(B) and VP2(C). The phylogenetic tree was constructed by using maximum likelihood (ML) with MEGA 7.01 software (http://www.megasoftware.net). Bootstrap values were calculated with 1000 replicates. The number on each branch indicates bootstrap values. The strains identified in the phylogenetic tree are indicated by GenBank accession numbers and breeds and strains. The seven P-PARV4 strains and eight O-PARV4 strains newly identified in the present study are indicated by " $\boldsymbol{\Delta}^{\text {" and " } \bullet ", ~}$ respectively. P-PARV4, O-PARV4 and B-PARV4 are shown in red, blue and green, respectively. Scale bar indicates nucleotide substitutions per site 
branches; 6 of the 8 new O-PARV4 strains in ovines and Tibetan sheep were closely related to each other and formed a separate branch. However, the new O-PARV4 strains of MG365908/ovine/CHN/GS3 discovered in this study formed one branch with JF5004702/Sheep/HK-S26, and MG365910/Tibetan sheep/CHN/GS4 formed one branch with JF5004699/Sheep/HK-S01, JF5004700/Sheep/ HK-S04 and JF5004701/Sheep/HK-S07 that was isolated from sheep in Hong Kong, China (Fig. 1). The phylogenetic tree of the NS1, VP1 and VP2 genes indicated that MG365908/ovine/CHN/GS3 and MG365910/Tibetan sheep/CHN/GS4 were also located in the same separate subcluster (Fig. 2). The identity analyses for full-length VP2 genomes of O-PARV4 revealed $98.84-100.00 \%$ sequence identity among the 7 strains and the previously reported strain, which for P-PARV4 was 98.60-99.28\%.

To identify the amino acid changes in the NS1, VP1 and VP2 proteins of the O-PARV4 and P-PARV4 strains in this study, we aligned the deduced amino acid sequences of the full-length NS1, VP1 and VP2 sequences with JF504699/Sheep/HK-S01 and JQ177079/Porcine/ $\mathrm{SH} 2$, respectively. As shown in Fig. 3, the mutation could be observed in the NS1, VP1 and VP2 sequences of most O-PARV4 and P-PARV4 strains. However, there were no amino acid substitutions in the NS1 sequences of the two strains of O-PARV4 (MG365908/ovine/CHN/ GS3 and MG365910/Tibetan sheep/CHN/GS4), and the numbers of mutations were also lower in the VP1 and VP2 sequences. Furthermore, the number of mutations of deduced amino acids in the NS1, VP1 and VP2 sequences of O-PARV4 was higher than that in P-PARV4, and no recombination was found in these strains (Fig. 3).

\section{Discussion}

The comprehensive investigation in our present study demonstrated a widespread distribution of PARV4-related viruses in livestock in the provinces surrounding the Qinghai-Tibet Plateau in China. All these data indicated that the prevalence of P-PARV4 and O-PARV4 differed among the different animals, ages and areas, which is consistent with the results of the PARV4 prevalence in domestic animals in other countries and the provinces in China [4, 5, 14-16]. Therefore, detection and further analysis of PARV4-related viruses from different geographic areas will be helpful for understanding the worldwide distribution and heterogeneity of PARV4-related viruses in animals and their potential zoonotic potential.

Similar to yaks, Tibetan pigs and Tibetan sheep have evolved over thousands of years as one of the unique and indigenous breeds in China. By living in cold and harsh environments on plateaus for a long time, the special livestock on the Qinghai-Tibet Plateau have undergone

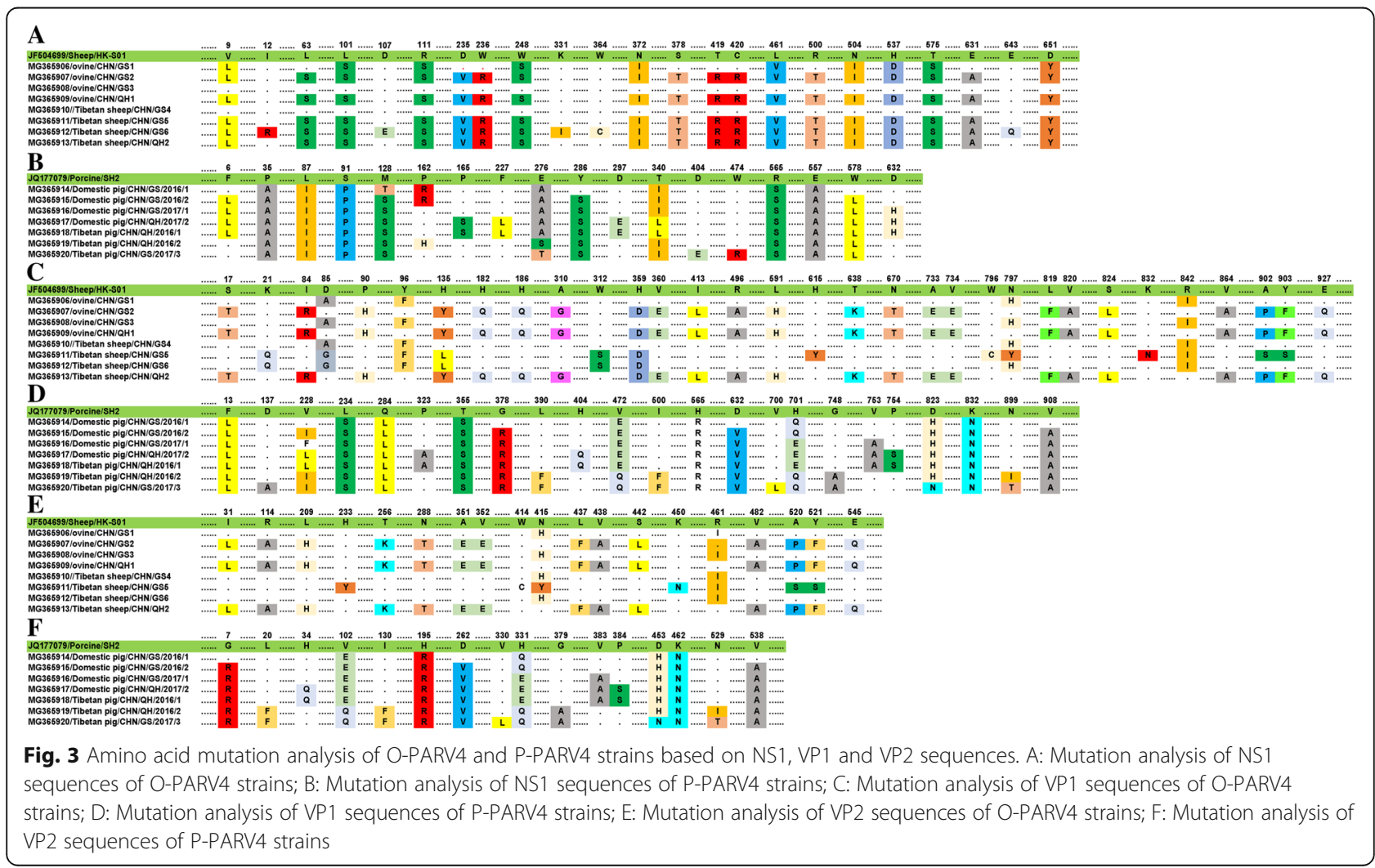


specific selection to enrich disease resistance-related gene categories in their genome $[6,7,17,18]$. The results of this study showed a lower prevalence of P-PARV4 in Tibetan pigs and O-PARV4 in Tibetan sheep than in domestic pigs and ovines. The same phenomenon was also observed for B-PARV4 infections in yaks [3]. The cold and harsh environment might be another factor that affects the transmission and infection of pathogens and results in a lower prevalence of PARV4-related viruses in livestock on the Qinghai-Tibet Plateau. Additionally, other pathogens infecting Tibetan pigs and yaks also provide information that the special livestock on the Qinghai-Tibet Plateau show striking physiological differences from lowland piglets, such as HEV infection in yak [3] and SIV and HP-PRRSV in Tibetan pigs [11, 12]. Both of these studies show the effects of the environment on pathogen transmission and infection, and these factors should not be ignored in future studies, especially on the Qinghai-Tibet Plateau.

The most interesting data in this study were from the molecular characterization of PARV4-related viruses in livestock on the Qinghai-Tibet Plateau, which was confirmed by phylogenetic and identity analyses using 13 novel and 30 previously reported PARV4-related whole-genome sequences (Fig. 1), which were consistent with those of phylogenetic analyses based on full-length NS1, VP1, and VP2 sequences obtained by the maximum likelihood (ML) method (Fig. 2). Phylogenetic analysis showed that all of the current P-PARV4 strains in this study are located on one branch and are closely related to strain JQ177079/Porcine/SH2 discovered in Shanghai in eastern China [4], suggesting that they might have evolved from the same ancestors. For the O-PARV4 strains in this study, there are three separate subclusters in the phylogenetic tree based on whole-genome sequences of PARV4-related strains. A distant relation was also shown by a phylogenetic tree based on full-length NS1, VP1, and VP2 sequences for MG365908/ovine/CHN/GS3 and MG365910/Tibetan sheep/CHN/GS4. The nucleotide and amino acid sequence identities of NS1 in these two O-PARV4 strains to JF504699/Sheep/HK-S01 were 100\% (Fig. 3), and four and two amino acid mutations were found in VP1 and VP2 sequences of these two O-PARV4 strains, respectively. These results demonstrated that the O-PARV4 strain in Tibetan sheep and ovines was the same strain as JF504699/Sheep/HK-S01 and was transmitted from Hong Kong, which needs further study. In addition, the high number of amino acid changes in O-PARV4 strains indicated that the mutation possibility of O-PARV4 is higher than that in P-PARV4, and more studies are needed to confirm this finding.

\section{Conclusions}

Collectively, our study is the first to demonstrate the presence of P-PARV4 in Tibetan pigs and O-PARV4 in Tibetan sheep, which provides a better understanding of the molecular epidemiology, genetic diversity and phylogenetic relationships of PARV4-related viruses in livestock in China and other PARV4-related reference strains. Until now, no evidence of cross-species infection or transmission has been reported, and the detection and further analysis of PARV4-related viruses in livestock and other animals on the Qinghai-Tibet Plateau will help to clarify the source, circulation pattern, zoonotic potential and public health risk of this virus. The mechanisms of its pathogenicity, transmission, evolution and persistence also require urgent clarification.

\section{Additional files}

Additional file 1: Detection rates of ungulate tetraparvovirus 2 ( $P$ PARV4) in domestic pigs and Tibetan pigs, ungulate tetraparvovirus 4 (O-PARV4) in ovine and Tibetan sheep on the Qinghai-Tibetan Plateau, China. (PDF $102 \mathrm{~kb}$ )

Additional file 2: Primers used for detection and full-length genome amplification of P-PARV4 and O-PARV4 in different hosts. (PDF $102 \mathrm{~kb}$ )

\begin{abstract}
Abbreviations
B-PARV4: Ungulate tetraparvovirus 1; BVDV: Bovine viral diarrhoea virus; HEV: Hepatitis E virus; HP-PRRSV: Highly pathogenic porcine reproductive and respiratory syndrome virus; ICTV: International Committee on Taxonomy of Viruses; NS: Non-structural proteins; O-PARV4: Ungulate tetraparvovirus 4; ORFs: Open reading frames; P-PARV4: Ungulate tetraparvovirus 2; SIV: Swine influenza A virus; VP: Viral capsid proteins
\end{abstract}

\section{Acknowledgements}

We are very grateful to Shaobo Chen. (Gansu Animal Disease Prevention and Control Center, Lanzhou, China) for clinical sample collection.

\section{Funding}

This work was supported by the grants from the National Key Research and Development Program of China (No. 2017YFD0502200), the National Natural Science Foundation of China No. 31702311, the Natural Science Foundation of Gansu Province (No. 17JR5RA141), the Fuxi Foundation of Youth Talent at Gansu Agricultural University (No. Gaufx-02Y10). Scientific research foundation for the new Scholars, Gansu Agricultural university (No. GSAURCZX201701), Innovation foundation of the College of Veterinary Medicine, Gansu Agriculture University (No. JYCX-KX016).

\section{Availability of data and materials}

All data generated or analysed during this study are included in this published article and its Additional file.

\section{Authors' contributions}

YYP, YW, MW, ARB and SJY designed the study, analyzed the data, and wrote the paper; QZ, JZ and JM contributed to sample processing, viral DNA extraction, PCR and subcloning; GQX, LBW, JFF and YC provided essential reagents and helped to sample processing; SS (ARB professor) revised the paper. All authors read and approved the final manuscript.

\section{Ethics approval and consent to participate}

All animals were handled with the assistance of veterinarians from the local veterinary institute and animal health and epidemiology centre. All procedures in this study were approved in strict accordance with good animal practice following the guidelines of the Animal Care and Use 
Committee of Gansu Agricultural University and performed in accordance with animal welfare and ethics.

\section{Consent for publication}

Not applicable.

\section{Competing interests}

The authors declare that there are no competing interests regarding the publication of this paper.

\section{Publisher's Note}

Springer Nature remains neutral with regard to jurisdictional claims in published maps and institutional affiliations.

\section{Author details}

${ }^{1}$ Gansu Agricultural University, College of Veterinary Medicine, Lanzhou 730070, China. ${ }^{2}$ Anning Branch Lanzhou Genegal Hospital, Lanzhou 730070, China. ${ }^{3}$ University of South Bohemia in České Budějovice, Faculty of Fisheries and Protection of Waters, South Bohemian Research Center of Aquaculture and Biodiversity of Hydrocenoses, Research Institute of Fish Culture and Hydrobiology, Zátiší 728/II, 38925 Vodňany, Czech Republic. ${ }^{4}$ Animal Health and Epidemiology Center in Chengguang District, Lanzhou 73000, China. ${ }^{5}$ Department of Veterinary Medicine, Sindh Agriculture University Tandojam, Tandojam, Pakistan.

Received: 21 August 2018 Accepted: 22 April 2019

Published online: 02 May 2019

\section{References}

1. Lau SK, Woo PC, Tse H, Fu CT, Au WK, Chen XC, Tsoi HW, Tsang TH, Chan JS, Tsang DN, Li KS, Tse CW, Ng TK, Tsang OT, Zheng BJ, Tam S, Chan KH, Zhou B, Yuen KY. Identification of novel porcine and bovine parvoviruses closely related to human parvovirus 4. J Gen Virol. 2008;89:1840-8.

2. Csagola A, Zadori Z. Meszaros ITuboly T. detection of porcine parvovirus 2 (ungulate Tetraparvovirus 3) specific antibodies and examination of the serological profile of an infected swine herd. PLoS One. 2016;11:e0151036.

3. Xu F, Pan Y, Wang M, Wu X, Tian L, Baloch AR, Zeng Q. First detection of ungulate tetraparvovirus 1 (bovine hokovirus 1) in domestic yaks in northwestern China. Arch Virol. 2016;161:177-80.

4. Pan Y, Zeng Q, Zhu C, Hua X, Wang M, Pan K, Cui L. Frequency and characterization of porcine hokovirus ( $\mathrm{PHoV}$ ) in domestic pigs in eastern China. Arch Virol. 2012;157:1785-8.

5. Tse H, Tsoi HW, Teng JL, Chen XC, Liu H, Zhou B, Zheng BJ, Woo PC, Lau SK, Yuen KY. Discovery and genomic characterization of a novel ovine partetravirus and a new genotype of bovine partetravirus. PLoS One. 2011;6:e25619.

6. Li M, Tian S, Jin L, Zhou G, Li Y, Zhang Y, Wang T, Yeung CK, Chen L, Ma J, Zhang J, Jiang A, Li J, Zhou C, Zhang J, Liu Y, Sun X, Zhao H, Niu Z, Lou P, Xian L, Shen X, Liu S, Zhang S, Zhang M, Zhu L, Shuai S, Bai L, Tang G, Liu H, Jiang Y, Mai M, Xiao J, Wang X, Zhou Q, Wang Z, Stothard P, Xue M, Gao X, Luo Z, Gu Y, Zhu H, Hu X, Zhao Y, Plastow GS, Wang J, Jiang Z, Li K, Li N, Li X, Li R. Genomic analyses identify distinct patterns of selection in domesticated pigs and Tibetan wild boars. Nat Genet. 2013;45:1431-8

7. Megens HJ, Crooijmans RP, San Cristobal M, Hui X, Li NGroenen MA Biodiversity of pig breeds from China and Europe estimated from pooled DNA samples: differences in microsatellite variation between two areas of domestication. Genet Sel Evol. 2008;40:103-28.

8. Xu F, Pan Y, Baloch AR, Tian L, Wang M, Na W, Ding L, Zeng Q. Hepatitis E virus genotype 4 in yak, northwestern China. Emerg Infect Dis. 2014;20:2182-4.

9. Zhu W, Dong JB, Zhang J, Uchida K, Watanabe K, Goto Y, Haga T. Bos grunniens papillomavirus type 1: a novel deltapapillomavirus associated with fibropapilloma in yak. J Gen Virol. 2013;94:159-65.

10. Ma JG, Cong W, Zhang FH, Feng SY, Zhou DH, Wang YM, Zhu XQ, Yin H, Hu GX. Seroprevalence and risk factors of bovine viral diarrhoea virus (BVDV) infection in yaks (Bos grunniens) in Northwest China. Trop Anim Health Prod. 2016:48:1747-50

11. Liu GH, Zhou DH, Cong W, Zhang XX, Shi XC, Danba C, Huang SY, Zhu XQ. First report of seroprevalence of swine influenza a virus in Tibetan pigs in Tibet, China. Trop Anim Health Prod. 2014;46:257-9.
12. Fan B, Zhang H, Bai J, Liu X, Li Y, Wang X, Jiang P. Pathogenesis of highly pathogenic porcine reproductive and respiratory syndrome virus in Chinese Tibetan swine. Res Vet Sci. 2016;108:33-7.

13. Kumar S, Stecher G, Tamura K. MEGA7: Molecular evolutionary genetics analysis version 7.0 for bigger datasets. Mol Biol Evol. 2016;33:1870-4.

14. Adlhoch C, Kaiser M, Ellerbrok HPauli G. High prevalence of porcine Hokovirus in German wild boar populations. Virol J. 2010;7:171.

15. Souza CK, Streck AF, Goncalves KR, Pinto LD, Ravazzolo AP, de Barcellos DE, Canal CW. Phylogenetic characterization of the first ungulate tetraparvovirus 2 detected in pigs in Brazil. Braz J Microbiol. 2016;47:513-7.

16. Cadar D, Csagola A, Lorincz M, Tombacz K, Spinu MTuboly T. Distribution and genetic diversity of porcine hokovirus in wild boars. Arch Virol. 2011;156:2233-9.

17. Ma JG, Zhang XX, Zheng WB, Xu YT, Zhu XQ, Hu GX, Zhou DH. Seroprevalence and risk factors of bluetongue virus infection in Tibetan sheep and yaks in Tibetan plateau, China. Biomed Res Int. 2017;2017: 5139703.

18. Wang J, Blasdell KR. Yin HWalker PJ. A large-scale serological survey of Akabane virus infection in cattle, yak, sheep and goats in China. Vet Microbiol. 2017;207:7-12.

\section{Ready to submit your research? Choose BMC and benefit from:}

- fast, convenient online submission

- thorough peer review by experienced researchers in your field

- rapid publication on acceptance

- support for research data, including large and complex data types

- gold Open Access which fosters wider collaboration and increased citations

- maximum visibility for your research: over $100 \mathrm{M}$ website views per year

At BMC, research is always in progress.

Learn more biomedcentral.com/submissions 